\title{
Spatial Luminance Contrast Sensitivity: Effects of Surround
}

\author{
Youn Jin $\mathrm{Kim}^{1} *$ and Hong-suk $\mathrm{Kim}^{2}$ \\ ${ }^{1}$ Digital Media \& Communications Research Center, Samsung Electronics Company, Suwon 443-742, Korea \\ ${ }^{2}$ Department of Physics, Daejin University, Pocheon 487-711, Korea
}

(Received March 30, 2010 : revised April 26, 2010 : accepted April 26, 2010)

\begin{abstract}
This study examined the effects of surround luminance on the shape of the spatial luminance contrast sensitivity function (CSF). The reduction in brightness of uniform neutral patches shown on a computer controlled display screen is also assessed to explain the change of CSF shape. Consequently, a large amount of reduction in contrast sensitivity at middle spatial frequencies can be observed; however, the reduction is relatively small for low spatial frequencies. In general, the effect of surround luminance on the CSF appears similar to that of mean luminance. Reduced CSF responses result in less power of the filtered image; therefore, the stimulus should appear dimmer with a higher surround luminance.
\end{abstract}

Keywords: Surround luminance effects, Spatial luminance contrast sensitivity, Brightness

OCIS codes : (330.1800) Vision - contrast sensitivity; (330.5510) Psychophysics; (330.6100) Spatial discrimination; (330.7310) Vision

\section{INTRODUCTION}

The contrast sensitivity function (CSF) represents the value of the minimum contrast at each spatial frequency that is necessary for a visual system to distinguish a sinusoidal grating or a Gabor pattern over a range of spatial frequencies from a uniform field. Physiologically, both parvocellular (P) and magnocellular $(\mathrm{M})$ cells have receptive fields organized into two concentric antagonistic regions: a central region, which can be on-center or off-center and a surrounding region of opposite sense. This arrangement is common in vertebrates. The receptive fields of small bistratified cells appear to lack clear centre-surround organization [1]. The distributions of sensitivity within centre and surround mechanisms are usually represented by Gaussian profiles of a ganglion cell's receptive field. The spatial properties of the visual neurons are commonly inferred from a neuron's spatial modulation transfer function [2] or contrast sensitivity function [3] measured with grating patterns whose luminance is modulated sinusoidally. In practice, monochromatic patterns in which luminance varies sinusoidally in space are used. CSFs typically plot the reciprocal of the minimum contrast that is also referred to as threshold, and provide a measure of the spatial properties of contrast-detecting elements in the visual system [4]. It is believed that CSF is in fact the enve- lope of the sensitivity functions for collections of neural channels that subserve the detection and discrimination of spatial patterns [5-6].

The first measurement of luminance CSF for the human visual system (HVS) was reported by Schade [7] in 1956. The luminance CSF has been extensively studied over a variety of research fields - such as optics, physiology, psychology, vision and color science - and the same basic trends were observed. Luminance CSF exhibits a peak in contrast sensitivity at moderate spatial frequencies $(\sim 5.0$ cycles per degree; cpd) [4] and falls off at both lower and higher frequencies; thus, it generally shows band-pass characteristics. The fall-off in contrast sensitivity at higher spatial frequency can be explained by spatial limitations in the retinal mosaic of cone receptors. The reduction in contrast sensitivity at lower spatial frequencies requires further neural explanations [8]. Centre-surround receptive fields are one possible reason for this low-frequency fall-off [9].

The CIE technical committee (TC) 1-60 [10] has recently collected luminance CSF measurement data from the literature [11-14]. Those data were measured in different experimental contexts; for instance, Campbell and Robson [11] used Gabor patches and the others [12-14] used sinusoidal gratings. All of the data were normalized to unity at the maximum contrast sensitivity of each data set for a cross-

\footnotetext{
*Corresponding author: younj.kim@samsung.com
} 
comparison on a single plot. Consequently, they corresponded to one another and their trends are remarkably similar; therefore, they could be accurately fit by a single CSF model [14] in spite of the significant difference in conditions, methods and stimulus parameters.

The CSF model used was originally proposed by Barten [14] as a function of spatial frequency and dependent on field size (or viewing angle in degrees) and mean luminance of the sinusoidal grating stimulus. As the mean luminance of the sinusoidal grating stimulus is decreased, the following variations occur (See Figure 1). The contrast sensitivity at each spatial frequency decreases, and the maximum resolvable spatial frequency decreases. In addition, the shape of luminance CSF changes; the peaks in the functions shift toward lower spatial frequencies, broaden, and eventually disappear [15-17].

The wealth of data in the literature also reports a variety of changes in CSF shape with senescence, [18-22, 28] eccentricity [23-26, 29] and degree of adaptation to noise [27] in a given stimulus. Briefly, luminance CSFs for older subjects exhibit losses in contrast sensitivity at the higher frequencies, although much of the loss is attributed to optical factors. $[18,28]$ Sensitivity to the local contrast at the peripheral region can be measured by instructing the observer to fixate on a marker whilst the actual object is placed at some distance from the marker. The distance is usually expressed in an angular measure called 'eccentricity' and the contrast sensitivity is measured as a function of eccentricity. With increasing eccentricity, capillary coverage increases up to $40 \%$ [29]. Fairchild and Johnson [27] found the fact that the adapted luminance CSF relates to the reciprocal of the adapting stimulus' spatial frequency. However, surround

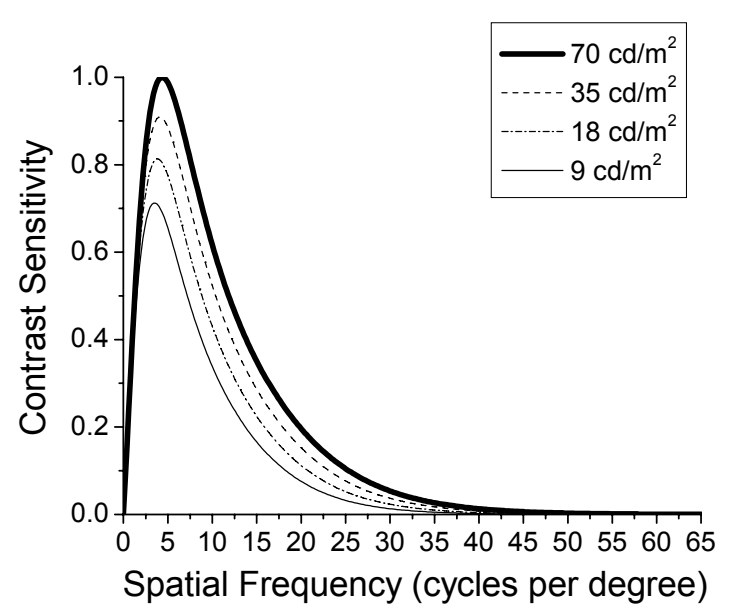

FIG. 1. Predicted CSF by Barten's model with various mean luminance levels for a field size of 5 degrees. As the mean luminance of the sinusoidal grating stimulus is decreased, contrast sensitivity at each spatial frequency decreases, and the maximum resolvable spatial frequency decreases as well. The peaks in the functions shift toward lower spatial frequencies and broaden. effects on the luminance CSF in the spatial frequency domain appears to be less well investigated thus far. Cox et al [30]. measured the effect of surround luminance on CSF and visual acuity using computer-generated sinusoidal gratings under surround levels up to $90 \mathrm{~cd} / \mathrm{m}^{2}$ for the purpose of ophthalmic practice in 1999. In consequence, reduced contrast sensitivity was measured under the highest surround luminance $\left(90 \mathrm{~cd} / \mathrm{m}^{2}\right)$ and the optimal surround level was found to be at $10 \sim 30 \%$ of mean luminance of a target stimulus. Precisely, contrast sensitivity increases when luminance of the surround increases from 0 to $10 \sim 20 \%$ of that of stimulus; however once the surround luminance exceeds the optimal level contrast sensitivity suddenly falls off.

Recently, portable display devices such as mobile phones and portable media players are viewed in a diverse range of surround luminance levels, and we usually experience images on a mobile phone display with a huge loss in contrast under bright outdoor viewing conditions. Ambient illumination and surround have been thought of as the first major factor among the mobile environmental considerations [31]; therefore, it is worth measuring the changes in luminance CSF shape under high brightness surrounds as a simulation of outdoor sunlight. In two psychophysical experiments we examined luminance CSFs under different surround luminance levels and the change in brightness of uniform neutral patches shown on a computer controlled display screen is observed. Specifically, Experiment 1 is conducted to measure the compound results of contrast threshold perception and physical contrast of a display resulting from the increase of ambient illumination. The former could be attributed to simultaneous lightness contrast [32] between stimuli on a display and surround luminance, so it may cause a change in CSF. The latter is usually decreased by viewing flare, the surface light reflections off the front of the monitor screen. In addition, in Experiment 2, a set of psychophysical assessments for variation in brightness are carried out to support and justify the surround effects on the CSF.

In this study, the modulation transfer function (MTF) of the display used is computed for each surround condition, then the MTF is divided into the results from Experiment 1 in order to deduce the display's resolution term, as well as the effects of viewing flare. Because resolution of the display device used may limit the detectable contrast sensitivity of a human observer, the display factor should be discounted. In an equation form, let $F(u, v)$ represent MTF of a display which comes from the Fourier transformed line spread function (LSF). If the image from the display is filtered by CSF denoted by $H(u, v)$, the Fourier transform of the output $\psi(u, v)$ can be given by $[14,55]$

$$
\psi(u, v)=H(u, v) F(u, v)
$$

where $\mathrm{u}$ and $\mathrm{v}$ are spatial frequency variables.

Therefore, CSF $H(u, v)$ can be estimated by deducing MTF $F(u, v)$ in a linear system (See Equation 2). Viewing 
flare is an additional luminance across all tonal levels from black to white, and increases only the zero frequency response. More detailed discussions follow in the Results section.

$$
H(u, v)=\psi(u, v) / F(u, v)
$$

\section{METHODS}

\subsection{Apparatus}

A 22.2-inc. Eizo ColorEdge221 liquid crystal display (LCD) was used to present experimental stimuli such as sinusoidal gratings and uniform neutral patches. Spatial resolution of the LCD is $1920 \times 1200$ pixels and the bit depth was 8 bits per channel. The maximum luminance producible is approximately $140 \mathrm{~cd} / \mathrm{m}^{2}$ in a dark room and the black level can reach $1 \mathrm{~cd} / \mathrm{m}^{2}$ due to the inherent leakage light problem of typical LCDs. The display was illuminated by using an EVL lighting Colourchanger 250 light source in a diagonal direction. The ambient illuminance levels could be adjusted by changing the distance between the display and light source. Two particular illuminance levels, i.e. 7000 and $32000 \mathrm{~lx}$, were achieved when the distance settings from the display were respectively 270 and $135 \mathrm{~cm}$. The white colored wall located behind the display was used as surround. In our previous works, [39-40] illuminance of a few real outdoor viewing situations was measured. The lower level (7000 $\mathrm{lx}$ ) is for simulating 'overcast' and the higher one (32000 lx) for 'bright' outdoor sunlight conditions. Note that the light source illuminates not only the surround region but also the display screen. The physical contrast loss, which can be caused by the light reflection from the screen (See Table 1), is deducted by using MTF of the display for each viewing condition. More details about this viewing flare compensation will be discussed later in the Results section.

Table 1 provides measured maximum and minimum luminance levels of the display for each viewing condition along with the viewing flare, absolute Michelson contrast (Mc), relative Mc to dark and surround luminance. Viewing flare can be estimated by the additional luminance increase due to the ambient illumination. As surround is changed from dark to overcast to bright, the mean of 8 evenly sampled luminance values across the surround wall behind the display increases from 0 to 1500 to $7000 \mathrm{~cd} / \mathrm{m}^{2}$. The amount of viewing flare also increases, so Mc levels (See Equation 3) are respectively decreased to 0.897 and 0.822 for overcast and bright as given in Table 1.

$$
\text { Michelson Contrast }=\left(\mathrm{L}_{\max }-\mathrm{L}_{\min }\right) /\left(\mathrm{L}_{\max }+\mathrm{L}_{\min }\right)
$$

where $L$ is luminance and maxima and minima are taken over the vertical position of the sinusoidal grating stimulus pattern.

The temporal stability of the light source was measured every 20 seconds continuously for 30 minutes from the cold start and the results are depicted in Figure 2. Crosses are measured data points for overcast and open circles are for bright. The illuminance level became stable after approximately 3 minutes for both cases. The stabilized illuminance values for the two lighting conditions were fluctuating around $6000 \sim 8000 \mathrm{~lx}$ for overcast and $31000 \sim 37000 \mathrm{~lx}$ for bright and their mean could be found near 7000 and $32000 \mathrm{~lx}$.

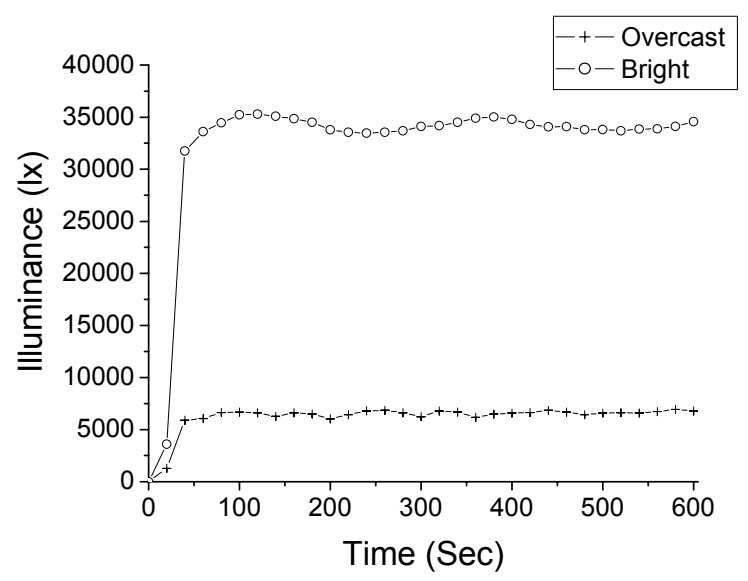

FIG. 2. Temporal illuminance measurement of the simulated outdoor sunlight using EVL lighting Colourchanger 250. The temporal stability of the light source was measured every 20 seconds continuously for 30 minutes from the cold start. Crosses are measured data points for overcast and open circles are for bright. The illuminance level became stable after approximately 3 minutes for both cases.

TABLE 1. Breakdown of each viewing condition

\begin{tabular}{l|c|c|c}
\hline \hline & Dark & Overcast & Bright \\
\hline $\mathrm{L}_{\max }\left(\mathrm{cd} / \mathrm{m}^{2}\right)$ & 140 & 147 & 154 \\
\hline $\mathrm{L}_{\min }\left(\mathrm{cd} / \mathrm{m}^{2}\right)$ & 1 & 8 & 15 \\
\hline Viewing Flare $\left(\mathrm{cd} / \mathrm{m}^{2}\right)$ & 0 & 7 & 14 \\
\hline Michelson Contrast $(\mathrm{Mc})$ & 0.986 & 0.897 & 0.828 \\
\hline Relative Mc to Dark & 1 & 1500 & 0.840 \\
\hline Surround luminance $\left(\mathrm{cd} / \mathrm{m}^{2}\right)$ & 0 & & 7000 \\
\hline
\end{tabular}




\subsection{Experiment 1: Compound Results of Contrast Thre- shold Perception and Physical Contrast Variation}

Experiment 1 is conducted to measure compound results of contrast threshold perception and physical contrast reduction caused by increase of ambient illumination. The former is affected by the level of surround luminance and the latter relates to the amount of viewing flare that was provided in Table 1. A sinusoidal grating pattern, of which contrast modulation gradually varies, is displayed on the display. Along the vertical axis of the screen, contrast becomes the highest in the bottom and lowest in the top of the pattern as can be seen in Figure 3. This sinusoidal grating pattern $(\mathbf{Q})$ was produced by means of the product of a non-linear gradient function along the vertical axis (M) and a one-dimensional sinusoidal function of spatial frequency across the horizontal axis $(\mathbf{F})$. In practice, those functions can be discretely sampled and expressed by

$$
\mathbf{Q}=\mathbf{M F}^{\mathrm{T}}
$$

where $\mathbf{F}^{\mathrm{T}}$ denotes the transpose of $\mathbf{F}$.

The compound effects of contrast threshold perception and physical contrast were measured at 11 spatial frequencies: $1,2,3,4,5,6,7,13,23,32$ and 65 cpd. The first 7 spatial frequencies (1 to $7 \mathrm{cpd}$ ) are sampled at the low spatial frequency area with steps of $1 \mathrm{cpd}$ in order to accurately measure the peak sensitivity and the sharp fall-off of CSF. Two middle spatial frequencies, 13 and $23 \mathrm{cpd}$, where the gradual fall-off after the peak can be observed, are also selected. The highest spatial frequency sampled in this study is $65 \mathrm{cpd}$ for predicting the maximum resolvable frequency.

In total, 6 observers (4 females and 2 males) participated in Experiment 1 and their ages ranged from 26 to 38. They were required to identify vertical positions of the sinusoidal pattern, by double-clicking a wireless mouse, when the contrast became just distinguishable. This experimental technique emulates a method suggested by Kitaguchi and MacDonald in 2006 [41]. We developed a software using Microsoft foundation class in Visual $\mathrm{C}+6.0$ to display sinusoidal patterns, to read the coordinates of double-clicked vertical position by the observer and to calculate the contrast. Technically, con-

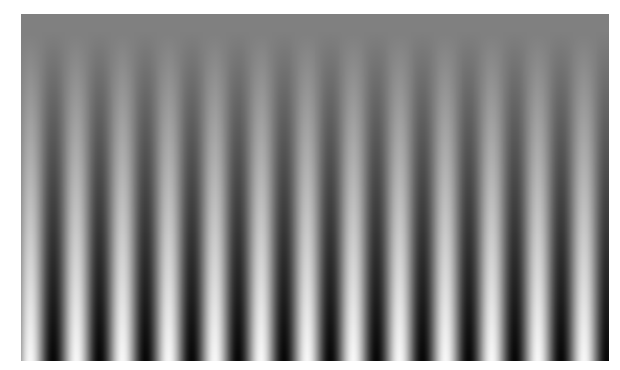

FIG. 3. Example of sinusoidal grating stimulus. Along the vertical axis of the screen, contrast becomes the highest in the bottom and lowest in the top of the pattern. trast can be defined as Michelson contrast (See Equation 3) and it is usually converted into a sensitivity unit that is the reciprocal of the contrast threshold as given in Equation 5 .

$$
\text { Sensitivity }=1 / \text { Threshold }
$$

Each sinusoidal pattern is displayed on the LCD monitor in a random order. Under the dark surround condition, the procedure was repeated 5 times and the results were averaged to obtain contrast threshold values. The same procedure was also applied for the other viewing conditions: overcast and bright. The sequence of these psychophysical sessions for the viewing conditions was also randomly decided for each observer. In order to assure maximum observer adaptation to the viewing condition including the LCD monitor white point and ambient illumination level, observers were given 30 seconds adaptation period [42] prior to each session. Specifically, observers were instructed to stare at a full white patch displayed on the LCD monitor screen under a certain ambient illumination. The distance between an observer and the LCD was set to be $3 \mathrm{~m}$ in order to minimize the quantization error of the 8-bit display used. The total number of psychophysical assessments collected for data analysis was $990(11$ stimuli $\times 5$ repeats $\times 6$ observers $\times 3$ viewing conditions).

\subsection{Experiment 2: Magnitude Estimation of Brightness}

Experiment 2 aims to measure the change in brightness [43] of a series of neutral colors shown on an LCD under varied ambient illumination levels and to find out whether the brightness change can affect the contrast threshold perception of human observers. The brightness / lightness distinction may not always be clear to subjects [59-61]. Lightness means perceived reflectance as a surface property, while brightness is even more ambiguously defined as the perceived luminance of a light source or subjective correlate of luminance [61]. We decided to adopt brightness because all of the test stimuli used are shown on a monitor rather than reflective colors.

Nine neutral patches were uniformly sampled across a 8-bit RGB scale from 0 to 255 with steps of 51 and each of the neutral colors was displayed at a time on the whole LCD screen. Five observers ( 2 females and 3 males) participated in this experiment in total and their ages ranged from 26 to 38. The apparent brightness of a full white patch displayed on the LCD monitor screen of which the RGB values are $(255,255,255)$ was assigned as an arbitrary brightness magnitude value of 100 . Prior to the brightness estimations, observers were required to memorize the white patch on the monitor in a dark room and judge a brightness ratio of each of the rest of test neutral colors at a time not only under dark but also under the other two ambient illumination conditions: overcast and bright. Observers were given the following written-instruction. "Please estimate the level of perceived brightness according to the reference patch whose perceived brightness is assigned as 100." Each observer 
repeated all judgments five times in a random order and their mean opinion scores (MOS) [44] were collected for data analysis.

The sequence of the experiment for those ambient illumination conditions was also randomly decided for each observer and a mid-gray of which RGB value is $(128,128$, 128) was shown to the observer as a transient patch whilst changing stimulus. The transient patch is usually displayed to prevent any illusions while the scene is changed. In the field of image quality, this illusion artifact is referred to as image sticking [45]. In order to assure maximum observer adaptation to the viewing condition including the LCD monitor white point and ambient illumination level, observers were given 30 seconds adaptation period [42] prior to each session. They were allowed to look back into the reference white patch under the dark viewing condition, but re-adaptations were performed when the viewing condition was altered. The total number of psychophysical assessments used for data analysis was $675(9$ stimuli $\times 5$ repeats $\times 5$ observers $\times 3$ viewing conditions).

\subsection{Statistical Analysis for Observer Variation}

Variation between observers was evaluated in terms of three test methods: ITU-R BT 500-10 [44], a modified version of coefficient of variance (CV) [46] and the Pearson correlation. First, ITU-R BT 500-10 method [44] rejects observations which are statistically incoherent with the other observers and show unusual peakedness of the probability distribution of a real-valued random variable. It should be ascertained whether the distribution of an observer's data is normal, using the Kurtosis test. Second, CV [46] is often used as a measure of the 'observer accuracy' which represents the mean discrepancy of a set of psychophysical data obtained from a panel of observers from their mean value. This term has been widely used in color appearance and difference studies $[46,58]$ and usage of it was also verified in image quality studies [39, 56-57]. The original $\mathrm{CV}$ is a normalized measure of dispersion for a repeated measurement but was applied to measure the degree to which a set of data points varied in this study. The CV is normally displayed as a percentage and, for a perfect agreement between them, equals to 0 . Third, Pearson correlation reflects the degree of linearity in the relationship between a pair of variables (e.g. $x$ and $y$ ). It is defined to be the sum of the products of the standard score of the two variables divided by the number of degrees of freedom. When the variables are perfectly linearly related, their Pearson correlation is +1 .

\section{RESULTS}

\subsection{Observer Variation}

Performance of the observers who participated in Experiments 1 and 2 was evaluated using the three statistical test methods previously introduced. The results obtained are summarized in Table 2. Basically, all of the observations can be accepted by the ITU-R BT 500-10 method and CV values ranged from 19 to 25 in Experiment 1 which can be within the acceptable level for observer accuracy [39, 46, 56-57]. Even lower CV values were measured in Experiment 2 (13 $\sim 18$ ) because of the simplicity of the magnitude estimation technique. Pearson correlations for all of the assessments are larger than 0.98 , meaning strong linearity between the mean and each observation. Especially for Experiment 2, since brightness estimates are known for their subject variability, the individual data are also illustrated along with their mean for each viewing condition in later section.

\subsection{Compound Results of Contrast Threshold Percep- tion and Physical Contrast}

In Experiment 1, the compound results of contrast threshold perception and physical contrast loss were achieved. They resulted from the increase of ambient illumination level causing both increase of surround luminance and viewing flare. Equation 5 was used to convert the measured data into sensitivity units, denoted as psi $(\psi)$, in Equations 1 and 2. Figure 4 depicts those $\psi$ data for the three viewing conditions. Every data point was normalized so that the maximum value obtained in dark (288) was unity, and adjacent data are linearly connected. Consequently, as the viewing condition changed from dark to overcast to bright, the data moved toward zero in general. The shape of all of the three plots appears typical band-pass and the spatial frequency where the maximum contrast sensitivity occurred was moved toward a lower frequency, i.e. from 5 to $4 \mathrm{cpd}$. The compound effects of surround luminance and viewing flare on the contrast threshold perception and physical contrast loss seem to be similar to that of mean luminance as previously

TABLE 2. Observer variation test results

\begin{tabular}{c|c|c|c|c}
\hline \hline \multirow{2}{*}{ Experiment } & Method & Dark & Overcast & Bright \\
\hline \multirow{3}{*}{1} & ITU-R BT 500-10 & All passed & All passed & All passed \\
\cline { 2 - 5 } & $C V$ & 20 & 19 & 0.994 \\
\cline { 2 - 5 } & $r$ & 0.983 & All passed & 18 \\
\hline \multirow{2}{*}{2} & ITU-R BT 500-10 & All passed & 13 & 17 \\
\cline { 2 - 5 } & $C V$ & 0.993 & 0.994 & 0.991 \\
\cline { 2 - 5 } & $r$ & & & \\
\hline
\end{tabular}




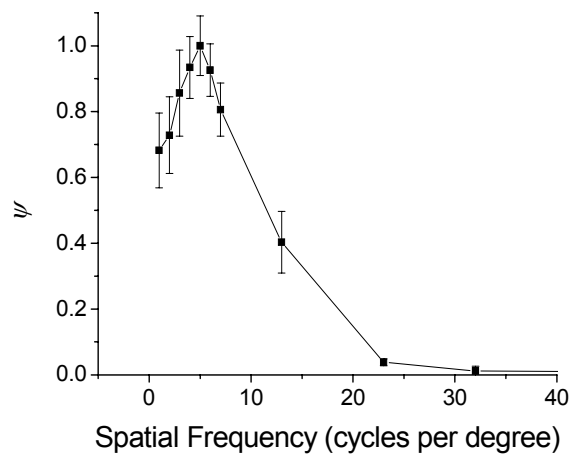

(a) Dark

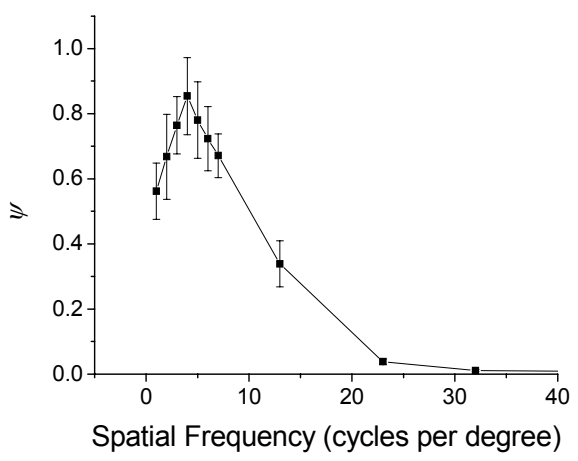

(b) Overcast

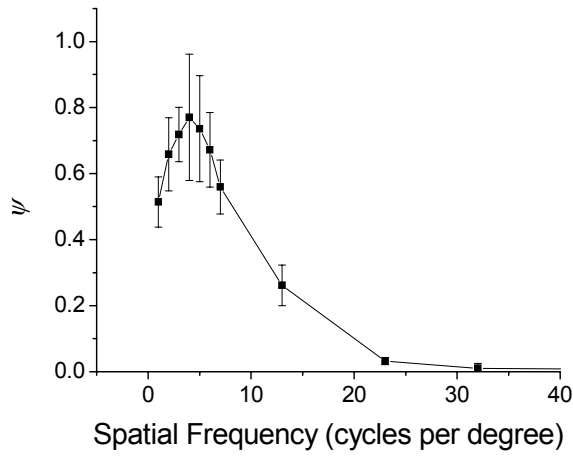

(c) Bright

FIG. 4. Measured data from Experiment 1 under 3 different ambient illumination conditions with linear interpolation for (a) dark (b) overcast and (c) bright. As the viewing condition changes from dark to overcast to bright, the data moved toward zero in general. The shape of the plots appears typical band-pass and the spatial frequency where the maximum contrast sensitivity occurred was moved toward a lower frequency, i.e. from 5 to $4 \mathrm{cpd}$.

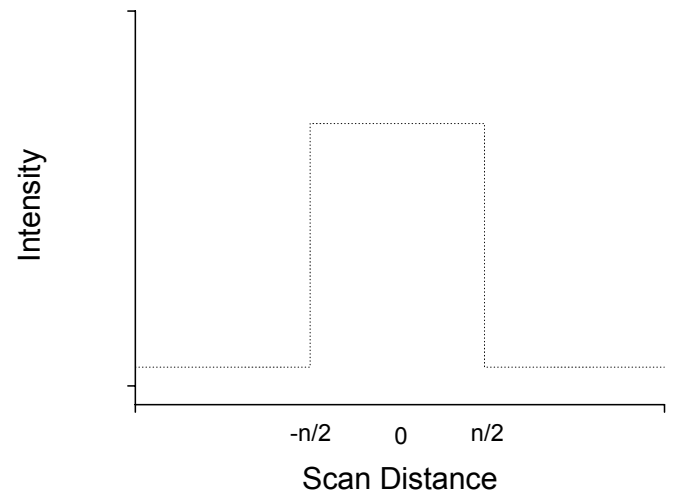

(a)

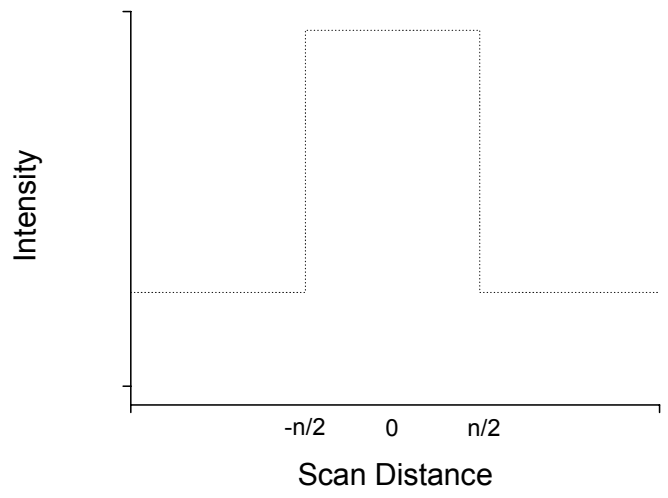

(b)

FIG. 5. (a) The original PSF and (b) viewing flare added PSF. Viewing flare is an additional luminance across the whole tonal levels from black to white and increases the zero frequency response only.

reported by the wealth of data in the literature as discussed in the Introduction section. Error bars represent standard errors that can be defined as standard deviation divided by square root of the number of observations.

\subsection{Deriving the Display MTF}

It is often assumed that the point spread function (PSF) of a majority of commercial LCD monitors is a rectangle function, $\operatorname{rect}(x),[47,62]$ because the shape of a single pixel in LCDs is rectangular as illustrated in Figure 5 (a). The rectangle function can be defined as

$$
\begin{array}{llll}
\operatorname{rect}(x)=1 & \text { if } & |x| \leq n / 2 \\
\operatorname{rect}(x)=0 & \text { if } & |x|>n / 2
\end{array}
$$

where $n$ is the size of a pixel of an LCD in visual angle [62].

Magnitude of the Fourier transform of the rectangle function can be expressed as shown in Equation 8.

$$
\begin{aligned}
\operatorname{MTF}(u) & =|\Im[\operatorname{rect}(x)]| \\
& =\left|\frac{\sin \pi n u}{\pi u}\right|
\end{aligned}
$$

Then Equation 8 is divided by n, because $\operatorname{MTF}(u)$ should be equal to 1 , so that the sinc function can be used as the MTF of the LCDs.

$$
\begin{aligned}
\operatorname{MTF}(u) & =\left|\frac{\sin \pi n u}{\pi n u}\right| \\
& =|\sin c(\pi n u)|
\end{aligned}
$$

where $\Im[$.$] denotes the Fourier transform of the argument.$

Viewing flare can be defined as the additional luminance due to surface reflections off the front of a display caused by ambient illumination. It boosts the PSF by a constant offset level as illustrated in Figure 5 (b); thus, only the zero frequency response (or $d c$ component) is increased and other frequency responses remain the same if the signal is 


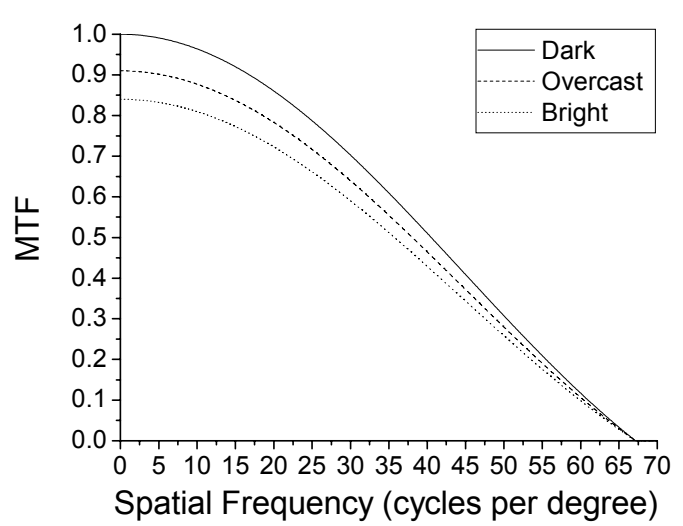

FIG. 6. MTF of the LCD used in this study and the approximated MTFs under two different levels of viewing flare. Single-pixel size of the LCD is set to be $0.00474^{\circ}$ in visual angle unit. The compensation factors $(\alpha)$ for viewing flare for the three viewing conditions are listed in Table 3.

transformed into the Fourier domain. When the MTF is normalized at the maximum, $\operatorname{MTF}(0)=1$ and $\operatorname{MTF}(u>0)$ is multiplied by a weighting factor $a$ for $u>0$ as shown by

$$
\begin{aligned}
\operatorname{MTF}_{i}(u) & =\alpha M T F_{0}(u) \\
& =\alpha|\sin c(\pi n u)|
\end{aligned}
$$

where $i$ represents the amount of viewing flare. As an example of this, $M T F_{0}$ shows the MTF for dark viewing condition so $M T F_{i}$ is the MTF for a viewing condition where the amount of viewing flare is $i \mathrm{~cd} / \mathrm{m}^{2}$. The weighting factor $a$ refers to the ratio of zero frequency response between $M T F_{0}(u)$ and $M T F_{i}(u)$ as given in Equation 11. Specifically, the mean value of the PSF can be used instead of calculating the zero frequency response of the MTF in the Fourier domain therefore $a$ values should be identical to the relative Michelson contrast to the dark viewing condition as can be expected (See Table 1).

$$
\alpha=\frac{M T F_{0}(0)}{M T F_{i}(0)}=\frac{\left(L_{M a x, 0}+L_{M i n, 0}\right) / 2}{\left(L_{M a x, i}+L_{M i n, i}\right) / 2}
$$

The estimated MTF of the LCD is presented in Figure 6 (See the solid line). Single- pixel size of the LCD is set to be $0.00474^{\circ}$ in visual angle units. The estimated MTFs for the higher illumination levels are shown in Figure 6 as well represented by dashed and dotted lines.

\subsection{Estimating CSF by Compensating for MTF}

As given in Equations 1 and 2 in the Introduction section, CSFs for the three viewing conditions can be estimated by dividing $\psi$ measured in Experiment 1 by the corresponding MTFs as illustrated in Figure 7. Data points for dark are linearly interpolated and represented by solid lines, with dashed lines for overcast and dotted lines for bright. As can

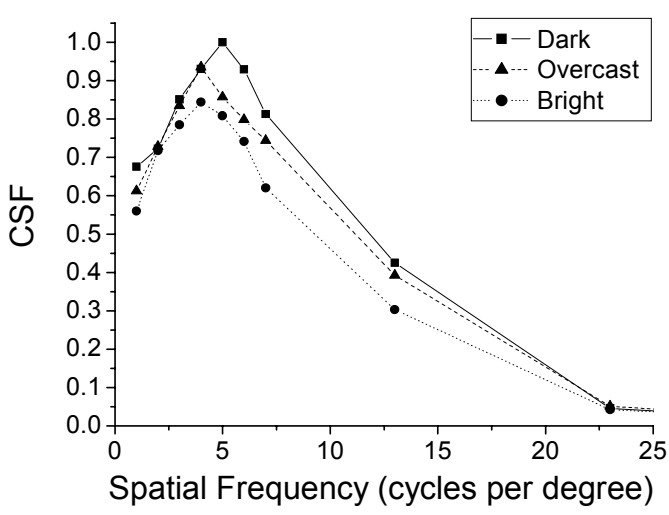

FIG. 7. Estimated CSF data points under 3 different surround luminance levels with linear interpolation. All of the three plots show band-pass characteristics and the peak spatial frequency for dark is $5 \mathrm{cpd}$ but moves to $4 \mathrm{cpd}$ for overcast and bright. A large amount of reduction in contrast sensitivity at middle frequency area $(4<u<13)$ can be observed; however, little reduction in contrast sensitivity is found for lower frequencies $(u<4)$.

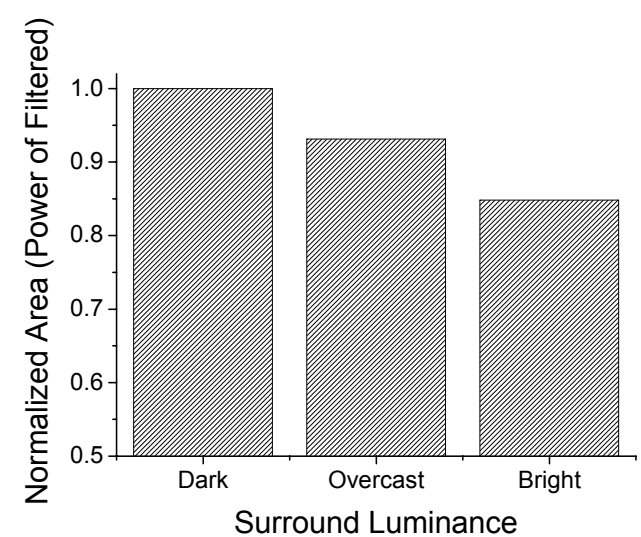

FIG. 8. Ratio of area of psi functions given in Figures 4 (a) through (c). The area of a function or a filter correlates to the power of a filtered image. As can be seen, about 15 and $23 \%$ of the loss in power occurred under overcast and bright, respectively due to the increase of ambient illumination.

be seen, they show band-pass characteristics and the peak contrast sensitivity for dark is observed at $5 \mathrm{cpd}$ but it moves to $4 \mathrm{cpd}$ for overcast and bright. The peak-shift appears more obvious compared to Figure 4. However, it is not easy to determine the significance of the peak-shift at the sampling frequency of $1 \mathrm{cpd}$. A large amount of reduction in contrast sensitivity at the middle frequency area $(4<u$ $<13$ ) can be observed; however, little reduction in contrast sensitivity is found for lower frequencies $(u<4)$. Because the MTF converges to zero at near the maximum spatial frequency we sampled (68 cpd) so contrast sensitivity at 65 cpd is not investigated in the current study due to the limited display resolution.

Figure 8 illustrates the ratio of the area covered by the 
three linearly interpolated plots previously shown in Figure 7. The area of a function or a filter correlates to the power of a filtered image. Area of each plot is normalized at the magnitude of the area for the dark viewing condition. As can be seen, about 7 and $15 \%$ of the loss in power occurred under overcast and bright, respectively due to the increase of surround luminance. The amount of power loss caused by the reduction in contrast sensitivity can be analogous to that of Michelson contrast reduction. As given in Table 1, Michelson contrast decrease reaches up to approximately 10 and $18 \%$ respectively for overcast and bright. It yields the fact that the amount of physical contrast reduction is larger than that of power loss in CSF. In order to statistically verify the surround luminance and spatial frequency effects on the shape in CSF, two-way analysis of variance (ANOVA) was performed with surround luminance and spatial frequency as independent variables and contrast sensitivity as the dependent variable. Significant effects could be found for both surround luminance and spatial frequency. Their $\mathrm{P}$ values were less than 0.0001 . A value of $P<0.05$ was considered to be statistically significant in this study.

Generally, the effect of surround luminance on the luminance CSF appears the similar as that of mean luminance, as previously discussed in Figure 1. Because CSF response correlates to the filtered light in the ocular media, smaller CSF responses across the spatial frequency domain result in less power of the filtered image; thus, less light can be perceived by the visual system. Therefore, the stimulus should appear darker under a higher surround luminance which can be verified through another set of experiments. The subsequent section discusses the results from Experiment 2.

\subsection{Change in Brightness Caused by Surround Lumi nance}

The mean perceived brightness magnitudes of the nine neutral colors for the 5 observers are drawn in Figure 9. The abscissa shows measured luminance of the neutral patches shown on an LCD. The ordinate represents their correspond- ing perceived brightness magnitudes. The filled circles indicate dark, empty circles for overcast and crosses for bright. Data points are linearly interpolated. As can be seen, all of the data points for overcast and bright are underneath data points for dark, which means that their perceived brightness is decreased in general, as the ambient illumination and surround luminance increase, in spite of the additional luminance increase by viewing flare. Similar results of brightness reduction between the surround and focal area can also be found in other works [48-49]. Since brightness estimates are known for their subject variability, the individual data are also illustrated along with their mean for each viewing condition in Figure 10. Filled circles show mean of the 5 observers and error bars show $95 \%$ confidential interval. As all of the observations were accepted by the three observer variability tests in Table 2, all of the brightness estimates follow the same trends. No particular outliers can be observed.

The precise relation between perceived brightness and

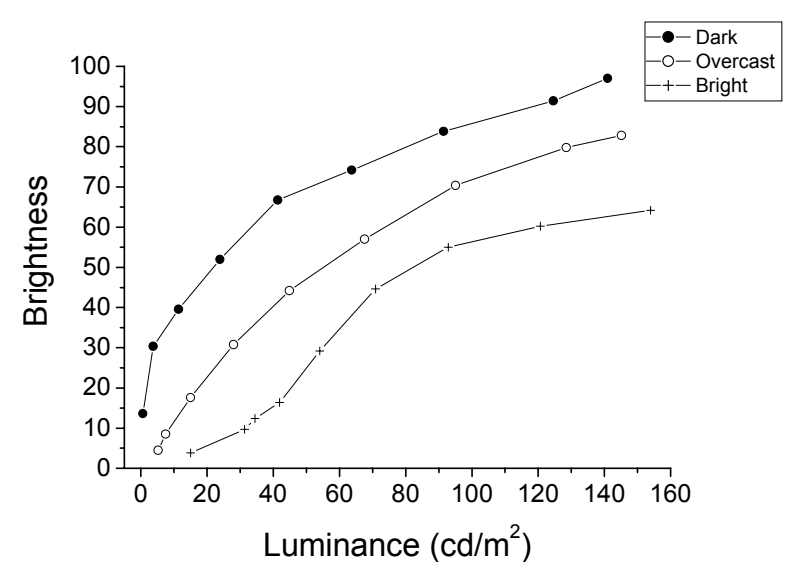

FIG. 9. Luminance vs. brightness under varied ambient illumination levels. All of the data points for overcast and bright are underneath data points for dark which means that their perceived brightness is decreased in general, as the ambient illumination and surround luminance increase in spite of the additional luminance increase by viewing flare.

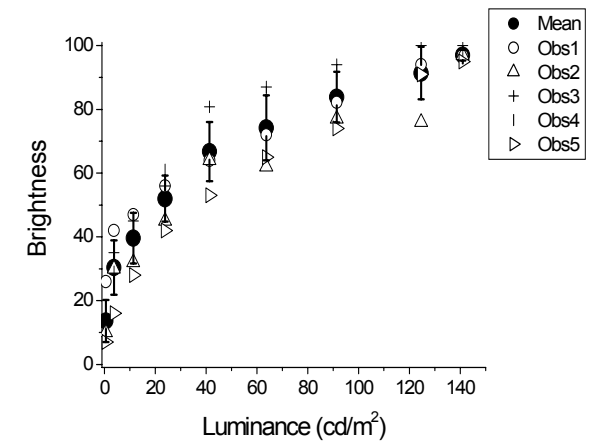

(a) Dark

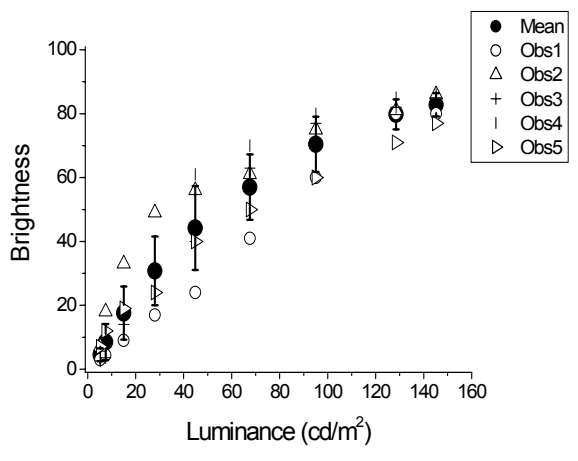

(b) Overcast

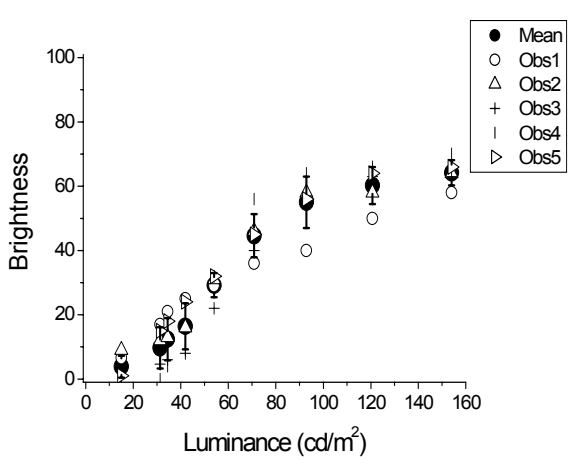

(c) Bright

FIG. 10. Individual brightness estimates for (a) dark (b) overcast and (c) bright. Brightness estimates are known for their subject variability but all of the brightness estimates follow the same trends. No particular outliers can be observed. Error bars show standard error. 
stimulus luminance has been extensively studied using reflective color samples. Traditionally, there are two most frequently cited explanations [50]. One of them is called the law of retinal stimulus. It is intuitively expected that, if the amount of light falling on a given stimulus is increased, the intensity of the retinal light image could be increased and the HVS could perceive its increased brightness. All of the stimuli should appear lighter with the aid of increased luminance from ambient illumination. The other most frequently cited explanation for the relation between perceived brightness and stimulus luminance is the law of brightness constancy [48, 50-52]. This phenomenon is based on neural processing after light rays pass through ocular media in the HVS. There are some examples that apparent brightness of visually perceived objects is relatively constant in real world: white snow always appears bright but black coal looks very dark over a range of illuminance, although the coal in the high illumination may actually reflect more intensity of light to the eye than does the snow at the low illumination. According to this theory, the relative brightness, with and without ambient illumination should be constant. However, our experimental results showed reduction in perceived brightness under ambient illumination and neither of the two traditional phenomena could predict this situation. One of the possible reasons for this is that the lighter surround makes the focal area appear darker and this phenomenon is referred to as simultaneous lightness contrast [32]. The neural contrast mechanism that makes the low-luminance areas appear darker in bright environments more than compensates for the reduced physical contrast caused by intraocular scatter [53-54].

\section{CONCLUSION}

This study examined the variation in shape of spatial luminance CSF under different surround luminance levels and reduction in brightness of uniform neutral patches shown on a computer controlled display screen is also assessed to explain change of CSF shape. Specificically, Experiment 1 was conducted to measure the compound results of contrast threshold perception and physical contrast decrease of a display resulting from the increase of ambient illumination. The former is found to be attributed to simultaneous lightness contrast [32] between stimuli on a display and surround luminance causes the change in CSF shape. The latter is usually decreased by the surface light reflections off of the front of the monitor screen referred to as viewing flare. Through a set of brightness magnitude estimations in Experiment 2, the surround luminance effects on the CSF and brightness reduction assumption could be justified. The viewing flare and display terms were successfully deduced by using MTF. Consequently, a large amount of reduction in contrast sensitivity at middle frequency area $(4<u<13)$ can be observed; however, little reduction in contrast sensitivity is found for lower frequencies $(u<4)$. They show band-pass characteristics and the spatial frequency where the maximum contrast sensitivity occurs moves from 5 to $4 \mathrm{cpd}$ when surround luminance increases from dark to overcast to bright. However, it is not quite easy to determine the significance of the peak-shift at the sampling frequency of $1 \mathrm{cpd}$. Generally, the effect of surround luminance on the luminance CSF appears the similar as that of mean luminance. Because CSF response can correlate to the filtered light in the ocular media, smaller CSF responses across the spatial frequency domain result in less power of the filtered image; thus, a smaller amount of light can be perceived by the visual system. Therefore, the stimulus should appear dimmer under a higher surround luminance. The power loss in CSF reaches up to 7 and $15 \%$ respectively for overcast and bright. Analogously, the Michelson contrast decrease was 10 and $18 \%$ for overcast and bright due to the fact that the amount of physical contrast reduction is larger than that of power loss in CSF. In order to evaluate the statistical significance of the surround luminance and spatial frequency effects on the shape in CSF, two-way ANOVA was performed and significant effects could be found for both parameters.

The results, which can be obtained from Experiments 1 and 2, are applicable to various purposes. Since CSFs have been widely used for evaluating image quality by predicting the perceptible differences between a pair of images [33-35, 55] surround luminance effects on CSF can be very useful for this application. Furthermore, the results can also be applied to simulate the appearance of a scene [36-37] and evaluate the visual performance of the eye [38]. It should be noted that only luminance CSF in fovea are considered in this paper and peripheral region CSF remains for future work. Chromatic CSFs in opponent color channels with varied surround luminance levels can be another interesting topic for a future study.

\section{REFERENCES}

1. D. M. Dacey and B. B. Lee, "The blue-on opponent pathway in the primate retina originates from a distinct bistratified ganglion cell," Nature 367, 731-735 (1994).

2. F. L. van Nes and M. A. Bouman, "Spatial modulation transfer in the human eye," J. Opt. Soc. Am. 57, 401-406 (1967).

3. C. Enroth-Cugell and J. G. Robson, "The contrast sensitivity of retinal ganglion cells of the cat," J. Physiol. 187, 517552 (1966).

4. F. W. Campbell and D. G. Green, "Optical and retinal factors affecting visual resolution,” J. Physiol. 181, 576-593 (1965).

5. O. Braddick, F. W. Campbell, and J. Atkinson, "Channels in vision: basic aspects," in Handbook of Sensory Physiology, R. Held, H. W. Leibowitz, and H.-L. Teuber, eds. (Springer-Verlag, New York, USA, 1978), vol. 8.

6. N. Graham, "Spatial-frequency channels in human vision: detecting edges without edge-detectors," in Visual Coding 
and Adaptability, C. S. Harris, ed. (Erlbaum, Hillsdale, NJ, USA, 1980).

7. O. H. Schade, "Optical and photoelectric analog of the eye,” J. Opt. Soc. Am. 46, $721-739$ (1956).

8. S. Westland, H. Owens, V. Cheung, and I. PatersonStephens, "Model of luminance contrast-sensitivity function for application to image assessment," Col. Res. Appl. 31, 315-319 (2006).

9. B. A. Wandell, Foundations of Vision (Sinauer Associates, Sunderland, MA, USA, 1995).

10. E. Martinez-Uriegas, Spatial and Temporal Problems of Colorimetry (CIE, Vienna, Switzerland, 2006), Chapter 3.

11. F. W. Campbell and J. G. Robson, "Application of Fourier analysis to the visibility of gratings," J. Physiol. 197, 551566 (1968).

12. A. B. Watson, "Visual detection of spatial contrast patterns: evaluation of five simple models," Opt. Exp. 6, 12-33 (2000).

13. E. Martinez-Uriegas, J. O. Larimer, J. Lubin, and J. Gille, "Evaluation of image compression artefacts with ViDEOS, a CAD system for LCD color display design and testing," Proc. SPIE 2411, 74-82 (1995).

14. P. G. J. Barten, Contrast Sensitivity of the Human Eye and Its Effects on Image Quality (SPIE Press, Bellingham, WA, USA, 1999).

15. A. M. Rohaly and G. Buchsbaum, "Global spatiochromatic mechanism accounting for luminance variations in contrast sensitivity functions," J. Opt. Soc. Am. A 6, 312-317 (1989).

16. A. S. Patel, "Spatial resolution by the human visual system," J. Opt. Soc. Am. 56, 689-694 (1966).

17. R. L. de Valois, H. Morgan, and D. M. Snodderly, "Psychophysical studies of monkey vision - III. Spatial luminance contrast sensitivity tests of macaque and human observers," Vision Res. 14, 75-81 (1974).

18. C. Owsley, R. Sekuler, and D. Siemsen, "Contrast sensitivity throughout adulthood," Vision Res. 23, 689-699 (1983).

19. U. Tulunay-Keesey, J. N. V. Hoever, and C. Terkla-McGrane, "Threshold and suprathreshold spatiotemporal response throughout adulthood," J. Opt. Soc. Am. A 5, 2191-2200 (1988).

20. K. E. Higgins, M. J. Jaffe, R. C. Caruso, and F. DeMonasterio, "Spatial contrast sensitivity: effects of age, test-retest, and psychophysical method," J. Opt. Soc. Am. A 5, 21732180 (1988).

21. A. M. Rohaly and C. Owsley, "Modeling the contrastsensitivity functions of older adults," J. Opt. Soc. Am. A 10, 1591-1599 (1993).

22. S. Pardhan, "Contrast sensitivity loss with aging: sampling efficiency and equivalent noise at different spatial frequencies," J. Opt. Soc. Am. A 21, 169-175 (2004).

23. J. Rovamo, V. Virsu, and R. Nasanen, "Cortical magnification factor predicts the photopic contrast sensitivity of peripheral vision," Nature 271, 54-56 (1978).

24. J. J. Koenderink, M. A. Bouman, A. E. B. de Mesquita, and S. Slappendale, "Perimetry of contrast detection thresholds of moving spatial sine wave patterns, Parts I. The near peripheral visual field (eccentricity 0-8)," J. Opt. Soc. Am. 68, 845-865 (1979).

25. M. J. Wright and A. Johnston, "Spatiotemporal contrast sensitivity and visual field locus," Vision Res. 23, 983-989 (1983).

26. A. Johnston, "Spatial scaling of central and peripheral contrast-sensitivity functions," J. Opt. Soc. Am. A 4, 1583-1593
(1987).

27. M. D. Fairchild and G. M. Johnson, "Measurement and modeling of adaptation to noise in image," J. Soc. Inf. Dis. 15, 639-647 (2007).

28. K. B. Burton, C. Owsley, and M. E. Sloane, "Aging and neural spatial contrast sensitivity: photopic vision," Vision Res. 33, 939-946 (1993).

29. D. M. Snodderly, R. S. Weinhaus, and J. C. Choi, "Neuralvascular relationships in central retina of macaque monkeys (Macaca fascicularis)," J. Neurosci. 12, 1169-1193 (1992).

30. M. J. Cox, J. H. Norma, and P. Norman, "The effect of surround luminance on measurements of contrast snesitivity," Ophthal. Physiol. Opt. 19, 401-414 (1999).

31. Z. Li, A. K. Bhomik, and P. J. Bos, "Introduction to mobile displays," in Mobile Displays Technology and Applications (Wiley, Chichester, UK, 2008).

32. S. Palmer, Vision Science: Photons to Phenomenology (MIT Press, Cambridge, MA, USA, 1999).

33. S. Daly, "The visible differences predictor: an algorithm for the assessment of image fidelity," in Digital Images and Human Vision, A. B. Watson, ed. (MIT Press, Cambridge, MA, USA, 1993).

34. X. M. Zhang and B. A. Wandell, "A spatial extension to CIELAB for digital color image reproduction," SID Digest 27, $731-734$ (1996).

35. Z. Wang and A. C. Bovik, Modern Image Quality Assessment (Morgan \& Claypool Publishers, NJ, USA, 2006).

36. E. Peli, "Test of a model of foveal vision by using simulations," J. Opt. Soc. Am. A 13, 1131-1138 (1996).

37. E. Peli, "Contrast sensitivity function and image discrimination,” J. Opt. Soc. Am. A 18, 283-293 (2001).

38. G. Yoon and D. R. Williams, "Visual performance after correcting the monochromatic and chromatic aberrations of the eye," J. Opt. Soc. Am. A 19, 266-275 (2002).

39. Y. J. Kim, M. R. Luo, W. Choe, H. S. Kim, S. O. Park, Y. Baek, P. Rhodes, S. Lee, and C. Kim, "Factors affecting the psychophysical image quality evaluation of mobile phone display: the case of transmissive LCD," J. Opt. Soc. Am. A 25, 2215-2222 (2008).

40. Y. J. Kim, M. R. Luo, P. Rhodes, S. Westland, W. Choe, S. Lee, S. Lee, Y. Kwak, D. Park, and C. Kim, "Imagecolour quality modelling under various surround conditions for a 2-inch mobile transmissive LCD," J. Soc. Inf. Dis. 15, 691-698 (2007).

41. S. Kitaguchi, L. MacDonald, and S. Westland, "Evaluating contrast sensitivity," Proc. SPIE 6057, 22-31 (2006).

42. M. D. Fairchild and L. Reniff, "Time-course of chromatic adaptation for color-appearance judgements," J. Opt. Soc. Am. A 12, 824-833 (1995).

43. B. Blakeslee, D. Reetz, and M. E. McCourt, "Comping to terms with lightness and brightness: effects of stimulus configuration and instructions on brightness and lightness judgments," J. Vision 8, 1-14 (2008).

44. ITU-R Rec. BT. 500-10, Methodology for the subjective assessment of the quality of television pictures, Geneva, Switzerland (2002).

45. H. J. Lee, D. W. Choi, E. Lee, S. Y. Kim, M. Shin, S. A. Yang, S. B. Lee, H. Y. Lee, and B. H. Berkeley, "Image sticking methods for OLED TV applications," in Proc. IMID (Ilsan, Korea, Oct. 2009), pp. 1077-1080. 
46. M. R. Luo, G. Cui, and C. Li, "Uniform colour spaces based on CIECAM02 colour appearance model," Col. Res. Appl. 31, 320-330 (2006).

47. P. G. J. Barten, "Resolution of liquid-crystal displays," SID Digest 22, 772-775 (1991).

48. H. Wallach, "Brightness constancy and the nature of achromatic colors," J. Exptl. Psychol. 38, 310-324 (1948).

49. E. G. Heinemann, "Simultaneous brightness induction as a function of inducing and test-field luminances," J. Exptl. Psychol. 50, 89-96 (1955).

50. D. Jameson and L. M. Hurvich, "Complexities of perceived brightness," Science 133, 174-179 (1961).

51. R. S. Woodworth and H. Schlosberg, Experimental Psychology (Holt, New York, USA, 1954).

52. D. Jameson and L. M. Hurvich, "Perceived color and its dependence on focal, surrounding, and preceding stimulus variables," J. Opt. Soc. Am. 49, 890-898 (1959).

53. W. A. Stiehl, J. J. McCann, and R. L. Savoy, "Influence of intraocular scattered light on lightness-scaling experiments," J. Opt. Soc. Am. 73, 1143-1148 (1983).

54. G. Westheimer and J. Liang, "Influence of ocular light scatter on the eye's optical performance," J. Opt. Soc. Am. A 12, 1417-1424 (1995).

55. P. G. Barten, "Evaluation of subjective image quality with the square-root integral method," J. Opt. Soc. Am. A 7,
2024-2031 (1990).

56. Y. J. Kim, Y. Bang, and H. Choh, "Gradient approach to quantify the gradation smoothness for output media," J. Electron. Img. 19, 011012 (2010).

57. Y. J. Kim, Y. Bang, and H. Choh, "Measurement and modelling of vividness perception and observer preference for color laser printer quality,” J. Img. Sci. Tech. 54, 010501 (2010).

58. M. R. Luo, G. Cui, and B. Rigg, "The development of the CIE 2000 colour-difference formula: CIEDE 2000," Col. Res. Appl. 26, 340-350 (2001).

59. L. E. Arend and B. Spehar, "Lightness, brightness and brightness contrast: I. Illumination variation," Percept. Psychophys. 54, 446-456 (1993).

60. L. E. Arend and B. Spehar, "Lightness, brightness and brightness contrast: II. Reflectance variation," Percept. Psychophys. 54, 457-468 (1993).

61. M. E. Rudd and D. Popa, "Stevens's brightness law, contrast gain control, and edge integration in achromatic color perception: a unified model," J. Opt. Soc. Am. A 24, 27662782 (2007).

62. Q. Sun and M. D. Fairchild, "Image quality analysis for visible spectral imaging systems," J. Img. Sci. Tech. 48, 211 -221 (2004). 